\title{
The impact of clinical maternal chorioamnionitis on neurological and psychological sequelae in very-low-birth weight infants: a case-control study
}

Francesc Botet ${ }^{1, *}$, Josep Figueras ${ }^{1}$, Xavier CarbonellEstrany $^{1}$, Eduardo Narbona ${ }^{2}$ and the Castrillo Study Group $^{3}$

${ }^{1}$ Neonatology Service, Hospital Clínic de Barcelona, Universitat de Barcelona, Barcelona, Spain

${ }^{2}$ Hospital Clínico, Universidad de Granada, Granada, Spain

${ }^{3}$ Castrillo Study Group: M. José Párraga Quiles and Juana M. Guzmán, Hospital Reina Sofía, Córdoba; Ana Alarcón Allen, Hospital Sant Joan de Déu, Esplugues de Llobregat, Barcelona; Carmen González Armengod, Hospital Clínico, Valladolid; Ana Remesal Escalero, Hospital Universitario de Salamanca, Salamanca; María J. García García, Hospital San Pedro de Alcántara, Complejo Hospitalario de Cáceres, Cáceres; Emilio Álvaro Iglesias, Hospital de León, León; Roser Porta, Institut Universitari Dexeus, Barcelona; Rafael Reparaz, Complejo Hospitalario Juan Canalejo, A Coruña; Belén Colomer and José López Sastre, Hospital Central de Asturias, Oviedo; and José Martínez Orgado, Fundación Hospital Alcorcón, Madrid, Spain

\footnotetext{
Abstract weight (VLBW) infants. performed.

*Corresponding author:

Francesc Botet, MD, PhD

Neonatology Service

Hospital Clínic de Barcelona

Sabino de Arana 1

E-08028 Barcelona

Spain

Tel.: +34-93-2275600

Fax: + 34-93-2275605

E-mail: fbotet@clinic.ub.es
}

Aims: To assess the relationship between clinically maternal chorioamnionitis and outcome in preterm very-low-birth

Methods: An observational case-control study was conducted in the neonatology departments of 12 acute care teaching hospitals in Spain. Between January 2004 and December 2006, all consecutive VLBW ( $\leq 1500 \mathrm{~g}$ ) infants who were born to a mother with clinical chorioamnionitis were enrolled. The controls included infants who were born to mothers without chorioamnionitis, matched by gestational age, and immediately born after each index case. At a corrected age of 24 months, a neurological examination and a psychological assessment of the surviving children were
Results: Sixty-six of the newborn infants died; therefore, 262 infants from the original sample were available for the study. Follow-up data were obtained at a corrected age of 24 months from a total of 209 children (106 cases and 103 controls, $80 \%$ of the original sample size). Seventy children $(33.5 \%)$ were diagnosed with some type of sequelae. The following conditions were all more prevalent in infants born to mothers with chorioamnionitis in comparison to controls: low development quotient $(98.3 \pm 12.15$ vs. $95.9 \pm 15.64$; $\mathrm{P}=0.497)$, cerebral palsy ( $4.9 \%$ vs. $10.4 \%$; $\mathrm{P}=0.138)$, seizures $(1.0 \%$ vs. $3.8 \%$; $\mathrm{P}=0.369)$, and other neurological or sensorial sequelae $(32.0 \%$ vs. $34.9 \%$; $\mathrm{P}=0.611)$.

Conclusions: After controlling for gestational age, the study population demonstrated that the neurological outcomes in infants at a corrected age of 24 months was not worsened by chorioamnionitis.

Keywords: chorioamnionitis; fetal membranes; follow-up; infant; premature; premature birth; premature rupture.

\section{Introduction}

Infection and inflammation are often related to preterm birth. Clinical, histological, and microbiological chorioamnionitis are clear examples of infection and inflammation that are related to birth and preterm birth dynamics. The greatest risk of histological chorioamnionitis occurs when delivery takes place at a gestational age of 23-24 weeks (79\%) and gradually decreases until 34 weeks (11\%) [9]. In recent years, significant attention has focused on the impact of antenatal inflammation on both short-term outcomes and long-term sequelae in preterm infants. In addition to severe acute morbidity, including early-onset sepsis, necrotizing enterocolitis, and lung disease $[3,10]$, preterm infants have a considerably increased risk of developing intraventricular hemorrhage and periventricular leukomalacia. These complications, in turn, are strong predictors of mental retardation and cerebral palsy $[6,17,18]$. On the basis of a known association between infection and very preterm birth, some authors proposed that chorioamnionitis plays a causal role not only in the induction of delivery but also in the fetal inflammatory response syndrome, which might cause acute morbidity and long-term sequelae $[6,12]$. Several studies have examined the effects of clinical maternal chorioamnionitis and histological chorioamnionitis on neonatal and neuro-developmental outcomes in preterm babies [8, 13, 14, 16]; however, the interpretation of these studies is confounded by gestational 
age, which was not adjusted in the analyses. Therefore, we designed a multi-center, case-control study in which cases and controls were matched by gestational age to assess the relationship between maternal clinical chorioamnionitis and neonatal outcomes at two-year follow-up in very-low-birth weight (VLBW) preterm infants. The neonatal results were recently published [2].

\section{Methods}

Between January 2004 and December 2006, the neonatology departments at 12 acute care, university-affiliated hospitals throughout Spain participated in this observational, prospective, and case-controlled study. The cases consisted of consecutive preterm infants who weighed $<1500 \mathrm{~g}$ and born to a mother with a history of clinical chorioamnionitis. The controls included infants born to a mother without a history of chorioamnionitis, matched by gestational age, and immediately delivered after the corresponding study case. Gestational age was determined by ultrasound examination at 8-10 weeks. The diagnoses of clinical chorioamnionitis in pregnant women who presented with premature labor or premature rupture of the membranes was based on the presence of uterine tenderness, fever $\left(>38^{\circ} \mathrm{C}\right)$, leukocytosis $\left(>12,000\right.$ cells $\left./ \mathrm{mm}^{3}\right)$, and increased serum concentrations of C-reactive protein (CRP) $(>30 \mathrm{mg} / \mathrm{L})$. $\mathrm{CRP}$ was determined at the time of patient care. A diagnosis of chorioamnionitis was made based on the presence of one clinical criterion (fever or tenderness) and at least one laboratory finding. Infants with chromosomal or congenital anomalies were excluded from the study. The study was approved by the institutional review board of each participating center, and written informed consent for data collection was obtained from the parents. The inclusion of cases and controls in the study did not involve any intervention. Infants received individualised, standard care in accordance with the current clinical guidelines.

A study protocol was designed in which the following data were prospectively recorded: maternal age, premature labor (persistent uterine contraction, morphological changes in the cervix), premature rupture of the membranes (PROM; determined by clinical or biochemical evidence of a loss of amniotic fluid), antibiotic treatment [used in 112 cases $(88.5 \%)$ and 46 controls (52.3\%)], antenatal steroid therapy (at least a single course of betamethasone), cesarean delivery, multiple gestations, and whether or not the birth occurred in a tertiary care center. Antibiotic treatment was started when PROM was diagnosed and continued for five days or until delivery. Newborn data were collected for the following characteristics: gestational age, sex, birth weight, intrauterine growth restriction (IUGR) (below the $10^{\text {th }}$ percentile using race- and sex-specific growth charts), the need for resuscitation with endotracheal intubation, and Apgar score at 5 min. Neonatal morbidity and mortality data included the presence of the following: respiratory distress syndrome, patent ductus arteriosus (and surgical correction), necrotizing enterocolitis (and surgical treatment), seizures, early-onset sepsis, late-onset sepsis, fungal sepsis, intraventricular hemorrhage, periventricular leukomalacia, bronchopulmonary dysplasia (BPD) or the requirement of oxygen at 28 days, chronic lung disease or the requirement of oxygen at a corrected age of 36 weeks of gestation, retinopathy of prematurity of grade $>2$ (and laser treatment), death, and survival without chronic lung disease. The length of the hospital stay was also recorded.

At a corrected age of 24 months, a systematic neurological examination and a psychological assessment using a psychometric test were performed on the surviving children (Bayley II or Brunet
Lezine). Data on weight, length, and head circumference in centimetres were measured at the time of assessment. Neuromotor functions (walking, sitting, using hands, and head control), the presence of seizures, vision (normal, the need for corrective lenses, no vision or only light with one eye, or no vision or only light with both eyes), auditory function (sensorineural or conductive hearing loss or need for hearing aids), difficulty in communication (not understanding words, signs, or gestures), and difficulty in expression were evaluated.

The Kolmogorov-Smirnov test was used to evaluate the distribution of data. Normally distributed data are expressed as mean and standard deviation (SD), and non-normally distributed data are expressed as median and interquartile $\left(25^{\text {th }}-75^{\text {th }}\right.$ percentile) range. Categorical variables are expressed as absolute numbers and percentages. Differences between cases and controls were assessed using the Mann-Whitney $U$-test or a one-way analysis of variance (ANOVA) for continuous variables and the $\chi^{2}$-test for categorical variables. SPSS, version 12.0 (SPSS Inc., Chicago, IL, USA), was used for the statistical analyses, where in a $\mathrm{P}<0.05$ was considered statistically significant.

\section{Results}

Sixty-six of the newly-born infants died; therefore, 262 infants from the original sample were available for followup at two-year. Follow-up data were obtained at the corrected age of two years from a total of 209 children (106 cases and 103 controls), which is equivalent to $80 \%$ of the original sample size. Seventy children were diagnosed with some type of sequelae $(33.5 \%)$.

To examine the comparability of groups, the characteristics of both are summarized in Table 1. As expected, mothers with chorioamnionitis received antibiotic therapy more often. The groups are not strictly comparable since in the study group a greater number were born in a university-affiliated hospital, fewer were part of a multiple gestation, fewer births occurred by cesarean section, the average Apgar score was lower, and neonatal resuscitation with intubation was done more frequently. These represent a greater risk of perinatal asphyxia in the group without chorioamnionitis, which would predispose these infants to further sequelae; however, the sample is too small to interpret the effect of perinatal asphyxia.

A total of 66 infants died during the perinatal period, specifically 28 in the chorioamnionitis group and 38 in the control group [2]. No infants died during the first two years of life. Follow-up data were available for 209 infants, specifically 103 from the study group and 106 from the control group. Fifty-three children were lost to follow-up; their perinatal characteristics are summarized in Table 2. A proper analysis of outcomes should be conducted using the entire cohort of patients and should not exclude patients who died or were lost to follow-up. Table 3 depicts the perinatal and neonatal data for the 119 infants who were not followed (66 dead and 53 lost to follow-up) and compares these cases (61) to the controls (58). As expected, mothers in the chorioamnionitis group received more antibiotic treatment, and the infants had a worse health status at birth (low Apgar scores at 1 and $5 \mathrm{~min}$ after birth, requiring endotracheal intubation 
Table 1 Perinatal characteristics of the study group and the control group.

\begin{tabular}{|c|c|c|c|}
\hline & Study $(n=103)$ & Control $(n=106)$ & P-value \\
\hline Corrected age (months) & $24.7 \pm 2.33$ & $25.1 \pm 2.68$ & $0.168 \mathrm{NS}^{*}$ \\
\hline Birth weight $(\mathrm{g})$ & $1109 \pm 236$ & $1083 \pm 211$ & $0.384 \mathrm{NS}$ \\
\hline Gestational age (weeks) & $28.5 \pm 1.99$ & $28.9 \pm 1.55$ & $0.086 \mathrm{NS}$ \\
\hline Apgar $1 \mathrm{~min}$ & $6.0 \pm 2.07$ & $6.8 \pm 1.91$ & 0.002 \\
\hline Apgar $5 \mathrm{~min}$ & $7.9 \pm 1.24$ & $8.5 \pm 1.34$ & $<0.001$ \\
\hline IUGR & $9(8.7 \%)$ & $17(16.0 \%)$ & $0.110 \mathrm{NS}$ \\
\hline Male & $54(52.4 \%)$ & $56(52.8 \%)$ & $0.953 \mathrm{NS}$ \\
\hline Multiple birth & $22(21.4 \%)$ & $36(34.0 \%)$ & 0.042 \\
\hline Not born in a tertiary care center & $4(3.9 \%)$ & $12(11.3 \%)$ & 0.043 \\
\hline No antenatal corticoids & $8(7.8 \%)$ & $14(13.9 \%)$ & $0.161 \mathrm{NS}$ \\
\hline Antibiotic treatment & $73(93.6 \%)$ & $32(54.2 \%)$ & $<0.001$ \\
\hline Cesarean & $57(55.3 \%)$ & $75(71.4 \%)$ & 0.016 \\
\hline Intubation resuscitation & $46(44.7 \%)$ & $33(31.4 \%)$ & 0.049 \\
\hline Hyaline membrane disease & $57(55.3 \%)$ & $71(67.0 \%)$ & $0.084 \mathrm{NS}$ \\
\hline Persistent ductus arteriosus & $30(29.4 \%)$ & $36(34.3 \%)$ & $0.452 \mathrm{NS}$ \\
\hline Necrotizing enterocolitis & $6(5.9 \%)$ & $9(8.6 \%)$ & $0.456 \mathrm{NS}$ \\
\hline Intraventricular hemorrhage & $18(17.5 \%)$ & $23(21.9 \%)$ & $0.422 \mathrm{NS}$ \\
\hline Grades III or IV & $4(3.9 \%)$ & $7(6.7 \%)$ & $0.370 \mathrm{NS}$ \\
\hline Periventricular leukomalacia & $19(19.0 \%)$ & $13(12.5 \%)$ & $0.202 \mathrm{NS}$ \\
\hline Retinopathy (> grade 2) & $15(15.2 \%)$ & $10(9.9 \%)$ & $0.262 \mathrm{NS}$ \\
\hline Early-onset sepsis & $5(4.9 \%)$ & $2(1.9 \%)$ & $0.277 \mathrm{NS} *$ \\
\hline Late-onset sepsis & $37(35.9 \%)$ & $38(36.9 \%)$ & $0.885 \mathrm{NS}$ \\
\hline BPD (oxygen at 28 days) & $37(35.9 \%)$ & $37(35.2 \%)$ & $0.918 \mathrm{NS}$ \\
\hline CLD (oxygen at 36 weeks PMA) & $12(12.0 \%)$ & $19(18.3 \%)$ & $0.212 \mathrm{NS}$ \\
\hline
\end{tabular}

*Using non-parametric or exact tests (Mann-Whitney $U$-test or Fisher's exact test, respectively). IUGR = intrauterine growth restriction, $\mathrm{BPD}=$ bronchopulmonary dysplasia, $\mathrm{CLD}=$ chronic lung disease, $\mathrm{PMA}=$ postmenstrual age.

for resuscitation) and more frequently presented with early sepsis. These results are similar to our previous data for the entire study group [2]. These conclusions were the same regardless of whether the analysis was performed separately for infants who died (66) vs. those lost to follow-up (53) or for the combined 119 infants.

The perinatal and neonatal medical histories of the infants with and without follow-up were similar, and, therefore, the sequelae are likely to be the same. Thus, it seems possible to extrapolate the conclusions from the sample to the entire population.

Follow-up results from the study group (chorioamnionitis) and control group at the corrected (or postmenstrual) age of two years are reported in Table 4.

\section{Discussion}

In this case-control study, premature infants born to mothers with clinical chorioamnionitis, were not significantly different than the control group at two-year of follow-up.

Several previous studies have related the presence of chorioamnionitis to neurological sequelae or cognitive disorders. Maternal chorioamnionitis may have lasting negative consequences for fetal brain development, resulting in long-term cognitive impairments even when major handicaps, such as cerebral palsy, are absent [15]. In contrast, other data indicated that chorioamnionitis alone should not be a causal factor in poor neurological outcomes. Neonatal morbidities, Mental Developmental Index (MDI) scores, Psychomotor
Developmental Index (PDI) scores, probabilities of normal MDI and PDI scores, and the prevalence of cerebral palsy were compared between the chorioamnionitis and control groups while controlling for gestational age, sex, and the maternal use of steroids and/or antibiotics. The chorioamnionitis group of 102 infants was compared to the 75 control infants. In VLBW infants, we observed similar MDI and PDI scores and similar risks of cerebral palsy in births affected by chorioamnionitis and in those unaffected [11]. Recent work has more strongly associated neurological sequelae with gestational age in comparison to the presence of chorioamnionitis. Neither surrogate indicators for, nor direct markers of, in utero exposure to acute inflammation were significantly associated with severe adverse outcomes; however, gestational age at delivery was significantly associated with outcome. Among children born between 23 and 32 weeks of gestation, neonatal complications, gestational age at delivery, and the caregiver's intelligence quotient, but not in utero exposure to acute inflammation, were factors associated with increased risks of severe adverse neurodevelopmental outcomes at six years of age [1].

Inflammation is the mechanism most widely implicated in the onset of preterm and term labor, as well as in fetal damage, Infection, and inflammation that act through biochemical mechanisms. In many cases, histological chorioamnionitis is asymptomatic or has few clinical manifestations, and pathogens may or may not be present in the amniotic fluid. Cytokine release-induced inflammation in an infectious process may have an important role in the genesis of premature labor, and inflammation is thought to play a patho- 
Table 2 Perinatal data for the populations with and without follow-up.

\begin{tabular}{|c|c|c|c|}
\hline & $\begin{array}{l}\text { Followed } \\
(\mathrm{n}=209)\end{array}$ & $\begin{array}{l}\text { Not followed } \\
(\mathrm{n}=53)\end{array}$ & P-value \\
\hline Birth weight $(\mathrm{g})$ & $1096 \pm 224$ & $1112 \pm 229$ & $0.649 \mathrm{NS}$ \\
\hline Gestational age (weeks) & $28.7 \pm 1.79$ & $28.5 \pm 1.75$ & $0.485 \mathrm{NS}^{*}$ \\
\hline Apgar $1 \mathrm{~min}$ & $6.4 \pm 2.03$ & $6.0 \pm 2.27$ & $0.283 \mathrm{NS}^{*}$ \\
\hline Apgar $5 \mathrm{~min}$ & $8.2 \pm 1.32$ & $8.4 \pm 1.60$ & $0.136 \mathrm{NS}^{*}$ \\
\hline IUGR & $26(12.4 \%)$ & $3(5.9 \%)$ & $0.182 \mathrm{NS}$ \\
\hline Male sex & $110(52.6 \%)$ & $22(43.1 \%)$ & $0.224 \mathrm{NS}$ \\
\hline Multiple birth & $58(27.8 \%)$ & $15(29.4 \%)$ & $0.813 \mathrm{NS}$ \\
\hline Not born in a tertiary care center & $16(7.7 \%)$ & $6(12.0 \%)$ & $0.394 \mathrm{NS}^{*}$ \\
\hline No antenatal corticoids & $22(10.8 \%)$ & $10(20.4 \%)$ & $0.069 \mathrm{NS}$ \\
\hline Antibiotic treatment & $105(76.6 \%)$ & $28(68.3 \%)$ & $0.281 \mathrm{NS}$ \\
\hline Cesarean & $132(63.5 \%)$ & $31(60.8 \%)$ & $0.723 \mathrm{NS}$ \\
\hline Intubation resuscitation & $79(38.0 \%)$ & $22(43.1 \%)$ & $0.499 \mathrm{NS}$ \\
\hline Hyaline membrane disease & $128(61.2 \%)$ & $28(56.0 \%)$ & $0.496 \mathrm{NS}$ \\
\hline Patent ductus arteriosus & $66(31.9 \%)$ & $20(40.8 \%)$ & $0.234 \mathrm{NS}$ \\
\hline Necrotizing enterocolitis & $15(7.2 \%)$ & 0 & $0.082 \mathrm{NS}^{*}$ \\
\hline Intraventricular hemorrhage & $41(19.7 \%)$ & $12(25.0 \%)$ & $0.415 \mathrm{NS}$ \\
\hline Grades III or IV & $11(5.3 \%)$ & $2(4.2 \%)$ & $0.750 \mathrm{NS}$ \\
\hline Periventricular leukomalacia & $32(15.7 \%)$ & $7(14.9 \%)$ & $0.892 \mathrm{NS}$ \\
\hline Retinopathy (> grade 1) & $25(12.5 \%)$ & $4(8.7 \%)$ & $0.471 \mathrm{NS}$ \\
\hline Early-onset sepsis & $7(3.4 \%)$ & $5(9.8 \%)$ & $0.064 \mathrm{NS}^{*}$ \\
\hline Late-onset sepsis & $75(36.4 \%)$ & $14(28.0 \%)$ & $0.263 \mathrm{NS}$ \\
\hline BPD (oxygen at 28 days) & $74(35.6 \%)$ & $17(34.7 \%)$ & $0.907 \mathrm{NS}$ \\
\hline CLD (oxygen at 36 weeks PMA) & $31(15.2 \%)$ & $10(20.8 \%)$ & $0.341 \mathrm{NS}$ \\
\hline
\end{tabular}

*Using non-parametric or exact tests (Mann-Whitney $U$-test or Fisher's exact test, respectively). IUGR=intrauterine growth restriction, $\mathrm{BPD}=$ bronchopulmonary dysplasia, $\mathrm{CLD}=$ chronic lung disease, $\mathrm{PMA}=$ postmenstrual age.

Table 3 Perinatal data for the 119 infants without follow-up (66 died and 53 lost to follow-up).

\begin{tabular}{llll}
\hline & Study $(\mathrm{n}=61)$ & Control $(\mathrm{n}=58)$ & P-value \\
\hline Birth weight (g) & $1008 \pm 251$ & $962 \pm 245$ & $0.316 \mathrm{NS}$ \\
Gestational age (weeks) & $27.5 \pm 1.96$ & $27.5 \pm 1.80$ & $0.726 \mathrm{NS} *$ \\
Apgar 1 min & $4.9 \pm 2.35$ & $5.9 \pm 2.31$ & $0.015^{*}$ \\
Apgar 5 min & $7.2 \pm 2.24$ & $8.0 \pm 1.76$ & $0.023^{*}$ \\
IUGR & $4(6.6 \%)$ & $7(12.5 \%)$ & $0.271 \mathrm{NS}$ \\
Male sex & $32(52.5 \%)$ & $34(60.7 \%)$ & $0.368 \mathrm{NS}$ \\
Multiple birth & $17(27.9 \%)$ & $19(33.9 \%)$ & $0.478 \mathrm{NS}$ \\
Not born in a tertiary care center & $4(6.7 \%)$ & $6(10.7 \%)$ & $0.438 \mathrm{NS} *$ \\
No antenatal corticoids & $11(18.0 \%)$ & $8(16.0 \%)$ & $0.777 \mathrm{NS}$ \\
Antibiotic treatment & $44(97.8 \%)$ & $18(54.5 \%)$ & 0.001 \\
Cesarean & $29(47.5 \%)$ & $32(57.1 \%)$ & $0.299 \mathrm{NS}$ \\
Intubation resuscitation & $40(66.7 \%)$ & $25(44.6 \%)$ & 0.017 \\
Hyaline membrane disease & $44(77.2 \%)$ & $38(69.1 \%)$ & $0.333 \mathrm{NS}$ \\
Patent ductus arteriosus & $22(39.3 \%)$ & $24(44.4 \%)$ & $0.583 \mathrm{NS}$ \\
Necrotizing enterocolitis & $9(15.8 \%)$ & $5(9.8 \%)$ & $0.355 \mathrm{NS} *$ \\
Intraventricular hemorrhage & $26(49.1 \%)$ & $22(42.3 \%)$ & $0.488 \mathrm{NS}$ \\
$\quad$ Grades III or IV & $13(24.5 \%)$ & $14(26.9 \%)$ & $0.779 \mathrm{NS}$ \\
Periventricular leukomalacia & $7(13.7 \%)$ & $8(16.0 \%)$ & $0.748 \mathrm{NS}$ \\
Retinopathy (> grade 1) & $3(12.0 \%)$ & $4(12.1 \%)$ & $0.989 \mathrm{NS}$ \\
Early-onset sepsis & $12(20.3 \%)$ & 0 & $<0.001^{*}$ \\
Late-onset sepsis & $22(40.0 \%)$ & $13(24.5 \%)$ & $0.086 \mathrm{NS}$ \\
BPD (oxygen at 28 days) & $12(42.9 \%)$ & $12(35.3 \%)$ & $0.543 \mathrm{NS}$ \\
CLD (oxygen at 36 weeks PMA) & $6(23.1 \%)$ & $8(25.0 \%)$ & $0.865 \mathrm{NS}$ \\
\hline
\end{tabular}

*Using non-parametric or exact tests (Mann-Whitney $U$-test or Fisher's exact test, respectively). IUGR =intrauterine growth restriction, $\mathrm{BPD}=$ bronchopulmonary dysplasia, $\mathrm{CLD}=$ chronic lung disease, PMA, postmenstrual age. 
Table 4 Follow-up results at two years.

\begin{tabular}{|c|c|c|c|}
\hline & Study $(\mathrm{n}=103)$ & Control $(n=106)$ & P-value \\
\hline Weight (g) & $11,337 \pm 1481$ & $10,869 \pm 1465$ & 0.024 \\
\hline Height (cm) & $85.4 \pm 4.14$ & $85.0 \pm 4.24$ & $0.523 \mathrm{NS}$ \\
\hline Head circumference $(\mathrm{cm})$ & $48.1 \pm 2.77$ & $47.9 \pm 1.94$ & $0.392 \mathrm{NS}^{*}$ \\
\hline Development quotient (\%) & $98.3 \pm 12.15$ & $95.9 \pm 15.64$ & 0.497 NS* \\
\hline Cerebral palsy $(\mathrm{CP})$ & $5(4.9 \%)$ & $11(10.4 \%)$ & $0.138 \mathrm{NS}$ \\
\hline Type of CP: & & & $0.487 \mathrm{NS}$ \\
\hline Diplegia & $2(40.0 \%)$ & $3(27.3 \%)$ & \\
\hline Hemiplegia & $3(60.0 \%)$ & $4(36.4 \%)$ & \\
\hline Tetraplegia & 0 & $3(27.3 \%)$ & \\
\hline Athetoid & 0 & $1(9.1 \%)$ & \\
\hline Seizures & $1(1.0 \%)$ & $4(3.8 \%)$ & $0.369 \mathrm{NS}^{*}$ \\
\hline Some sequelae & $33(32.0 \%)$ & $37(34.9 \%)$ & $0.611 \mathrm{NS}$ \\
\hline Walking & $6(5.8 \%)$ & $12(11.3 \%)$ & $0.157 \mathrm{NS}$ \\
\hline Sitting & 0 & $4(3.8 \%)$ & $0.122 \mathrm{NS}^{*}$ \\
\hline Hands & $3(2.9 \%)$ & $8(7.6 \%)$ & $0.134 \mathrm{NS}$ \\
\hline Head & 0 & $2(1.9 \%)$ & $0.498 \mathrm{NS} *$ \\
\hline Vision & $7(6.8 \%)$ & $11(10.6 \%)$ & $0.334 \mathrm{NS}$ \\
\hline Hearing & $10(9.8 \%)$ & $7(6.7 \%)$ & $0.411 \mathrm{NS}$ \\
\hline Communication & 0 & $5(4.7 \%)$ & $0.060 \mathrm{NS}^{*}$ \\
\hline Expression & $18(17.8 \%)$ & $22(21.4 \%)$ & $0.525 \mathrm{NS}$ \\
\hline
\end{tabular}

*Using non-parametric or exact tests (Mann-Whitney $U$-test or Fisher's exact test, respectively).

genic role in periventricular leukomalacia and cerebral palsy [12]. The use of antibiotics in pregnant women may change the course of chorioamnionitis, although it fails to eliminate the presence of pathogens in the amniotic fluid [5]. An early diagnosis of chorioamnionitis, together with the use of antibiotics and corticosteroids, may moderate the process of inflammation and improve neonatal and neurological longterm outcomes. The most beneficial treatment protocol for chorioamnionitis needs to be determined by a prospective study and then instituted. To date, the combination of maternal antibiotics and steroids appears most promising [7]. Currently, there does not appear to be a "silver bullet" that is capable of preventing unfavourable neuro-developmental outcomes in the setting of fetal and/or neonatal inflammatory response. Thus, there is clearly a need for more epidemiological studies using advanced techniques of laboratory research and neuro-imaging and sufficiently long follow-ups. It is hoped that these studies will identify pre- and postnatal inflammatory risk profiles by clarifying the relationship between inflammation markers and their expressions in fetal and neonatal circulation over time. Although such research will be complex, it is necessary for a successful development of new anti-inflammatory interventions in the newborn [4].

The findings of this study allow us to challenge the belief that inflammatory factors play a pathogenic role in the neurological injuries that are sustained by premature infants and their subsequent sequelae of cerebral palsy and cognitive disorders. This knowledge presents the possibility of improving the prognoses of infants who suffer from premature labor, PROM, and even maternal chorioamnionitis. It also supports a more conservative obstetric management of these situations because neonatal and long-term outcomes depend more on gestational age at the time of delivery than on the presence of chorioamnionitis.
This study sample is large enough and used sufficiently strict selection criteria to be considered reliable. Moreover, data collection was prospectively conducted. Both preterm populations were comparable in gestational age and weight. In the chorioamnionitis group, Apgar scores were lower and more infants were born in a tertiary care center, more mothers delivered vaginally, and there were more singleton gestations than in the control group. These differences were expected to be unfavourable for the study group, and, therefore, one would predict less favourable outcomes for these infants. There is no other study to-date that analyzes clinical chorioamnionitis using these characteristics. At follow-up, over $80 \%$ of the initial population was analyzed, which gives our study statistical reliability. Moreover, the analysis of neonatal pathology demonstrated that there were no differences between the children who were studied at follow-up and those who were lost; hence, we can extrapolate the results to the entire original study population.

A limitation in all studies of chorioamnionitis is the difficult diagnosis. Making a diagnosis of clinical chorioamnionitis is less precise than microbiological testing of the amniotic fluid or pathologic examination of the membranes, umbilical cord, and/or placenta. Unfortunately, there is no gold standard for making the diagnosis. Histological findings that are suggestive of chorioamnionitis in the placenta are common in premature labor and become more common with decreasing gestational age. The presence of pathogens in the amniotic fluid by itself is not diagnostic of chorioamnionitis. Clinical criteria can be confusing, but, if properly selected, they are useful in practice and allow prospective inclusion in studies like this.

In our study population, the only observed perinatal complications that were found to be associated with clinical maternal chorioamnionitis were lower Apgar scores, a more 
frequent need for resuscitation involving endotracheal intubation, and an increased incidence of early-onset sepsis; however, these complications and the presence of chorioamnionitis are not always accompanied by worse neurological outcomes at a corrected age of two years.

\section{References}

[1] Andrews WW, Oliver SP, Biasini F, Peralta-Carcelen AM, Rector R, Alriksson-Schmidt AI, et al. Early preterm birth: association between in utero exposure to acute inflammation and severe neurodevelopmental disability at 6 years of age. Am J Obstet Gynecol. 2008;198:466.e1-11.

[2] Botet F, Figueras J, Carbonell X, Arca G. Effect of maternal clinical chorioamnionitis on neonatal morbidity in very low birthweight infants: a case-control study. J Perinat Med. 2010; 38:269-73.

[3] Bracci R, Buonocuore G. Chorioamnionitis: a risk factor for fetal and neonatal morbidity. Biol Neonat. 2003;83:85-96.

[4] Cornette L. Perinatal inflammation and infection. Minerva Ginecol. 2005;57:411-21.

[5] Gomez R, Romero R, Nien JK, Medina L, Carstens M, Kim YM, et al. Antibiotic administration to patients with preterm premature rupture of membranes does not eradicate intraamniotic infection. J Matern Fetal Neonatal Med. 2007;20: 167-73.

[6] Hagberg H, Wennnerholm UB, Sävman K. Sequelae of chorioamnionitis. Curr Opin Infect Dis. 2002;15:301-6.

[7] Ledger WJ. Perinatal infections and fetal/neonatal brain injury. Curr Opin Obstet Gynecol. 2008;20:120-4.

[8] $\mathrm{Mu} \mathrm{SC}$, Lin CH, Chen YL, Ma HJ, Lee JS, Lin MI et al. Impact on neonatal outcome and anthropometric growth in very low birth weight infants with histological chorioamnionitis. J Formos Med Assoc. 2008;107:304-10.
[9] Muglia LJ, Katz M. The enigma of Spontaneous Preterm birth. N Engl J Med. 2010;362:529-35.

[10] Newton ER. Preterm labor, preterm premature rupture of membranes, and chorioamnionitis. Clin Perinat. 2005:32: 571-600.

[11] Polam S, Koons A, Anwar M, Shen-Schwarz S, Hegyi T. Effect of chorioamnionitis on neurodevelopmental outcome in preterm infants. Arch Pediatr Adolesc Med. 2005;159: $1032-5$.

[12] Romero R, Espinoza J, Gonçalves LF, Kusanovic JP, Friel L, Hassan S. The role of inflammation and infection in preterm birth. Semin Reprod Med. 2007;25:21-39.

[13] Soraisham AS, Singhal N, McMillan DD, Sauve RS, Lee SK. A multicenter study on the clinical outcome of chorioamnionitis in preterm infants. Am J Obstet Gynecol. 2009;200: 372e1-6.

[14] Suppiej A, Franzoi M, Vedovato S, Marucco A, Chiarelli S, Zanardo V. Neurodevelopmental outcome in preterm histological chorioamnionitis. Early Human Dev. 2009;85:187-9.

[15] Versland LB, Sommerfelt K, Elgen I. Maternal signs of chorioamnionitis: persistent cognitive impairment in low-birthweight children. Acta Paediatr. 2006;95:231-5.

[16] Willoughby RE, Nelson KB. Chorioamnionitis and brain injury. Clin Perinatol. 2002;29:603-21.

[17] Wu YW, Colford JM Jr. Chorioamnionitis as a risk factor for cerebral palsy: a meta analysis. J Am Med Assoc. 2000;284: 1417-24.

[18] Yoon BH, Park CW, Chaiworapongsa T. Intrauterine infection and the development of cerebral palsy. Br J Obstet Gynaecol. 2003;110(Suppl 20):124-7.

The authors stated that there no conflicts of interest regarding the publication of this article.

Received May 6, 2010. Revised August 26, 2010. Accepted September 24, 2010. Previously published online February 7, 2011. 